\title{
Probiotic Bacteriotherapy and Its Oral Health Perspective
}

\author{
Meghana Ajay Deshpande ${ }^{1}$, Sudhindra Baliga ${ }^{2}$, Sapna Randad 3 , Nilima Thosar ${ }^{4}$, Nilesh Rathi ${ }^{5}$ \\ ${ }^{1}$ Department of Paediatric and Preventive Dentistry, Sharad Pawar Dental College, Sawangi \\ (Meghe), Wardha, Maharashtra, India. ${ }^{2}$ Department of Paediatric and Preventive Dentistry, \\ Sharad Pawar Dental College, Sawangi (Meghe), Wardha, Maharashtra, India. ${ }^{3}$ Department of \\ Paediatric and Preventive Dentistry, Sharad Pawar Dental College, Sawangi (Meghe), Wardha, \\ Maharashtra, India. ${ }^{4}$ Department of Paediatric and Preventive Dentistry, Sharad Pawar Dental \\ College, Sawangi (Meghe), Wardha, Maharashtra, India. ${ }^{5}$ Department of Paediatric and Preventive \\ Dentistry, Sharad Pawar Dental College, Sawangi (Meghe), Wardha, Maharashtra, India.
}

\section{ABSTRACT}

\section{BACKGROUND}

As health professionals, we prescribe wide range of chemotherapeutics to the patients to control or to prevent the disease. When there is excessive use of antibiotics, it leads to imbalance between the beneficial and harmful microorganisms, making our body more susceptible to infections. Probiotics are living microorganisms which when administered in adequate amounts confer a health benefit on the host. They are living microorganisms added to food which beneficially affect the host by improving its intestinal microbial balance. Intestine's microbial colonization is determined by the maternal intestinal flora and surroundings. Oral cavity is a complex ecosystem which has rich and diverse microbiota. The change in environment may be due to illness, debility, behaviour, diet or medications. So, an obvious fact is that changes in this ecology may give rise to dental diseases. A slight change in environment promotes the potential pathogens gain competitive advantage under appropriate conditions. Then, the pathogens increase in great numbers to predispose a site to disease. In order to cure the disease, probiotic approach, may be used. In probiotic method, whole bacteria replacement therapy is given which may be very effective in eliminating the dangerous pathogens from the oral cavity. Different probiotics are provided in products such as in medicines, beverages, milk-based foods, dietary supplements, etc. This review highlights the effectiveness of probiotics in improving the oral health.

\section{KEY WORDS}

Probiotics, Lactobacillus, Bifidobacterium, Oral Health, Anticariogenic, Antiplaque
Corresponding Author:

Dr. Meghana Ajay Deshpande, Department of Paediatric and Preventive Dentistry, Sharad Pawar Dental College, Sawangi (Meghe), Wardha, Maharashtra, India.

E-mail: meghana.adeshpande@gmail.com

DOI: $10.14260 /$ jemds/2020/438

How to Cite This Article:

Deshpande MA, Baliga S, Randad S, et al. Probiotic bacteriotherapy and its oral health perspective. J Evolution Med Dent Sci 2020;9(34):2479-2483, $10.14260 / \mathrm{jemds} / 2020 / 538$

Submission 07-04-2020,

Peer Review 09-07-2020,

Acceptance 17-07-2020,

Published 24-08-2020.

Copyright (C) 2020 JEMDS. This is an open access article distributed under Creative Commons Attribution License [Attribution 4.0 International (CC BY 4.0)] 


\section{BACKGROUND}

We as physicians prescribe an array of modern medicines to patients as a part of therapy or to prevent the occurrence of disease. Antibiotics are one such type which are extensively used in all the specialties of medical sciences. With the availability of the effective and cheap antibiotics, it has become possible to efficiently treat infections and has thus helped to reduce the mortality rate. The increased use of antibiotics has led to a disproportion between the favourable and unfavourable microflora, thus making the individuals highly prone to infections. It also results in the development of resistance to various pathogens. For this reason, probiotics have become the recent topic of research.

Probiotics are living organisms which when administered in adequate amount provides beneficial effects to the host by improving intestinal microbial balance. Oral cavity is a complex ecosystem consisting of diverse microbiota. Any disturbance in the environment of the gut may affect the condition of oral cavity. So, it is high time to shift the paradigm from treatment to elimination of specific bacteria that alter the microbial environment with the help of probiotics.

\section{Evolution}

Probiotics are microorganisms which have been proved to provide health promoting effects in animals and humans. In ancient times, microorganisms were known to promote health. Fermented foods were considered beneficial as they were known to have therapeutic effects on the body. As learnt in microbiology, when harmless bacteria are allowed to culture in humans, they boost the immunity of the individuals. In 1877, Pasteur and his associate noted that anthrax bacilli growth the growth of anthrax bacilli was suppressed when cultured with commonly found bacilli (possibly E. coli). They remarked that this observation may perhaps justify the increasing expectations for therapeutics.(1) In 1907, Eli Metchnikoff while working at the Pasteur Institute, suggested that the strain of Lactobacillus bulgaricus, found in Bulgarian yoghurt can move pathological intestinal microbiota. The modification of the microbial flora of our bodies is possible due to the dependence of the harmful microbes on food.(2) By 1950s, the United States department of Agriculture, had given license for a probiotic product to be served as a therapeutic agent in the management of scour (an E. coli infection) amongst pigs.

In 1965, Lilley and Stillwell coined the term probiotics as an autonym to antibiotic, describing them as microbes which enhance the growth of beneficial microflora.(3) In 1974, Parker introduced probiotics in the diet of animals and suggested that probiotics can also be administered to improvise the intestinal flora. ${ }^{(4)}$ The presently used definition of probiotics put forward by the World Health Organization and by the Food and Agriculture Organization of the United States is "Live microorganisms which when administered in adequate amounts confer a health benefit on the host".

\section{Mechanism of Action}

A number of mechanisms have been suggested to contribute to the probiotic action. Firstly, the probiotics perform their work by competing for their resources and space with the pathogens present within the host. Secondly, they secrete by-products that cause harm to the pathogens but are harmless to the host. These beneficial bacteria produce Bacteriocin like inhibitory substances which are a protein chain that are used to inhibit the growth of pathogenic bacteria. Thirdly, the probiotics trigger the host's immune system to grid for battle against pathogenic bacteria. They achieve an increased resistance towards infectious diseases which stimulate T lymphocytes to replicate.

Probiotics improve the colonization resistance to gut pathogens by reinforcing the mucosal barrier and restoring normal gut micro - ecology after diarrhoea. They produce antimicrobial substances, balance $\mathrm{pH}$ by producing short chain fatty acids and lactic acids and increase macrophage activity. There is competitive inhibition for the bacterial adhesion sites and competition for nutrients. The probiotics are known to reduce constipation and cholesterol levels.

The antigen transport is increased in case of deficiency of intestinal micro-flora. They are also known to normalize the increased permeability and compete with the pathogens for the binding sites and available substrates.

\section{Selection of Probiotics}

There should be definite criteria for considering products safe for consumption. The probiotics should not have any toxic effects and be a non-pathogenic preparation. They should have a beneficial effect on the host. They should resist the gastrointestinal juice. They should have a decent shelf-life and the intestinal micro flora should be restored or substituted.

\section{Ideal Properties of Probiotics}

1. Probiotics must be amongst the naturally existing microorganisms in order to be able to survive in presence of acid and bile; during their transit through the intestine; adhere to the mucosal lining of the intestine; and decrease the harmful pathogens colonizing the gut.

2. They should play a role in the oral ecology by inhibiting a biofilm and protecting oral tissues from diseases.

3. They should have a cariostatic, antioxidant and antifungal activity.

4. They should provide immunostimulatory action.

5. They should increase the large bowel motility and stool mass.

6. They should reduce the risk of conditions such as infectious diarrhoea \& general intestinal malaise.

\section{Functions of Probiotic Agents}

1. Improving the absorption of vitamins and minerals and improving digestion.

2. Improving the absorption of calcium which is important for the prevention of osteoporosis.

3. Generating vitamin B complex

4. Supporting the host for the healthy functioning of the liver.

5. Stabilizing the bowel elimination difficulties and promoting uniformity. 
6. Avoiding intestinal tract infections such as candidiasis and peptic ulcer condition.

7. Alleviating the bloating and belching.

8. Assisting in cholesterol management.

9. Defending us against harmful bacteria, fungi and viruses.

\section{ROLE OF PROBIOTICS IN DIFFERENT CONDITIONS}

\section{Probiotics \& Diabetes Mellitus \& Obesity}

Metabolic balance in the body is preserved by microflora of the gut. Furet JP et al conducted a study (2010) on diabetes mellitus patients and discovered that they had less number of Faecalibacterium prausnitzii and more number of inflammatory markers.(5) Similar results were obtained when individuals with diabetes mellitus type II were compared with nondiabetic patients.(6) Schwiertz A et al (2010) observed that less number of Bifidobacterium species were found in obese individuals when compared with lean people.(7) These conclusions advocate that Bifidobacterium can lead to obesity and its related comorbidities.

\section{Probiotics-Allergy and Atopic Diseases of Children}

In children with atopic diseases, the intestinal microflora has a greater number of $S$. aureus and Clostridium compared to Enterococcus, Bifidobacterium and Bacteroides which are found in much lesser numbers.(8) With the increased awareness of beneficial microflora of the gut, considerable efforts have been made to evaluate the action of probiotics through clinical trials, in preventing and/or managing allergic diseases. M. Kalliom"aki et al conducted a randomized placebocontrolled trial, in which Lactobacillus GG was fed to infants with high risk and they witnessed a $50 \%$ decline in atopic eczema. ${ }^{(9)}$ Another study was conducted by H. Majamaa and E. Isolauri in Finland, in which a whey formula with L. rhamnosus or B. animalis sp. Lactis was administered to children for a period of 2 months and as a result, improvements in the skin conditions were observed.(10) Rosenfeldt $\mathrm{V}$ et al also reported similar curative results with $L$. rhamnosus plus $L$. reuteri preparations.(11)

\section{Probiotics and Cancer Prevention}

Klinder $\mathrm{A}$ et al conducted a study where rats and mice were administered with inulin and/or oligofructose and they witnessed a decrease in the genotoxicity of faecal water.(12) Another study was conducted by Bolognani F et al (2001) to evaluate their effect on aberrant crypt foci formation in rats, and they observed that Probiotics lessened the number of chemically induced precancerous lesions and stimulated defence functions.(13) Roller $\mathrm{M}$ et al also observed an increase in level of IL-10 and NK-cell activity when long term treatment was done with a prebiotic in infected rats.(14)

\section{Probiotics and Oral Health}

The humans' oral cavity has a dynamic ecosystem. Oral mucosa, which is in constant contact with the external environment, is easily colonized by microorganisms. The resident oral microflora is diverse with differing nutritional, atmospheric and physiochemical traits. Temperature, $\mathrm{pH}$, nutrient availability, saliva and gingival crevicular fluid are factors which mainly have effects on the oral cavity.(15-19) The oral cavity generally maintains a homeostasis with the host. Any disturbance in this physiological process, may lead to exogenous and endogenous infections, illnesses, debility, changes in diet and behaviour, and also allow few selected species to thrive. $(20,21)$ Oral health conditions usually associated with decreased levels of beneficial bacteria include gingivitis, thrush, cold sores, etc. With increase in the complexity of microflora, there is a need to innovate alternative solution to block pathogenesis of oral infections and thus, reduce them. Probiotics have proven to be effective in treating diseases related to the gut. Dental diseases stem from digestive problems. Hence, probiotics can be used as a supplement to treat oral diseases. A lot of the frozen bacteria do not become rejuvenated and they die once they reach the gut. Hence, highly concentrated probiotics can be administered into the oral cavity with very little loss in number.

Majority of the probiotic agents are dairy products which have high content of calcium in them. This is possibly a measure to prevent the demineralization of teeth. They may act as a protective shield, to keep the harmful microflora away and reside in space that might then be colonized by them. In order to establish a cariostatic effect, probiotics should adhere to the dental tissues and hence become a part of the biofilm to provide defence against the cariogenic bacteria.

Some of the suggested mechanisms of actions of probiotics in the oral cavity are ${ }^{(22)}$

1. Direct contact with dental plaque.

2. Contribution in attachment of oral microflora to proteins.

3. Effect on plaque foundation and its ecological unit by opposing and superseding with bacterial adherence.

4. Participation in breakdown of substrate and release of chemicals that inhibit oral microbiota.

\section{Indirect Probiotic Actions}

1. Modifying systemic immunity.

2. Effect on local immune function.

3. Effect on non-immunologic defence mechanisms.

4. Regulation of mucosal penetrability.

5. Probiotics as antioxidants.

6. Avoid plaque development by neutralizing the free electrons.

\section{Effects of Probiotics in Oral Cavity}

i. Cariostatic effect: Probiotics adhere to dental tissues and be a part of the biofilm to fight with cariogenic microorganisms. According to a study, the biofilm foundation was considerably decreased in supernatant form by the lactobacillus-containing probiotic preparations. A stronger and major inhibitory influence was found by the cell-free supernatants on biofilm foundation than their bacterial counterparts. ${ }^{(23)}$ Although there are decent numbers of studies suggesting anticarcinogenic effect in intestines, there are no reported 
studies regarding the effect of probiotics on precancerous and malignant conditions of the oral cavity. $(24,25)$

ii. Antiplaque effect: Probiotics adhere to hydroxyapatite and prevent stain \& plaque formation on teeth. Biological plaque control may exert beneficial effects by reducing the growth of P. gingivalis in subgingival sites. A study suggested that passive immunization with the use of egg yolk antibody against periodontal pathogens may be an effective treatment for periodontitis.(26)

iii. Antifungal effect: Elahi et al (2005) checked the pattern of colonization of L. acidophilus and L. fermentum in mice on the consumption of probiotic strains and witnessed a sudden deterioration in C. albicans count. Constant intake of probiotics significantly decreased the amount of fungal infection in the oral cavity, thus, maintaining the protective influence for a persistent period after the termination of application.(27) Although, the consumption of probiotics reduces the prevalence of oral candida but it is found to increase the risk of hyposalivation. Hence, further research needs to be carried out to rule out the potential risk of probiotics in reducing salivation.

iv. In periodontal diseases: Russian scientists have reported that probiotic containing Bifidobacterium species have successfully reduced gingival \& periodontal inflammation.(28) Koretz RL. et al (2009) conducted a study with $L$. reuteri and observed a decline in gingival bleeding and decrease in gingival inflammation.(29) Ojetti $\mathrm{V}$ et al (2009) conducted a randomized trial to evaluate the effectiveness of oral supplementation with L. reuteri in lactose intolerant patients and witnessed that lactobacilli flora inhabiting the gut inhibited the growth of Prevotella intermedia and Porphyromonas gingivalis up to $65 \%$ and $82 \%$, respectively.(30)

\section{Administration of Probiotics}

Various forms of delivering probiotic strains are suggested in numerous articles. Milk products enhanced with probiotics can be delivered perorally. However, with the purpose of prevention or management of oral diseases, precisely targeted applications, formulations or vehicles with measured release of probiotics might be desired.(27) Nasopharyngeal rinse, sodium chloride throat spray and pure bacterial pellets are some of the suggested vehicles for the administration of probiotics. C. Aglar et al (2006) conducted a study in which he evaluated the effects of two non-dairy administration techniques, a Life top straw (BioGaia AB, Stockholm, Sweden) and a lozenge on the efficiency of $L$. reuteri to decrease the number of $\mathrm{S}$. mutans. Both the modes of administration showed substantial decline in salivary S. mutans levels.(31) The latest development for prevention of caries is by administrating $L$. reuteri incorporated in chewing gum. When administered two times a day, it was advocated to control S. mutans colonization in the oral cavity.(31) However, it may can be concluded that the proper mode and amount of probiotics to be administered for the numerous oral health problems is still not defined.(32)

\section{Future Perspectives}

Further research on probiotics may help and assist in providing localized passive immunization against dental caries. In infants, the early mucosal colonization by E. coli causes the mucosal immune system to form specific antibodies and nonspecific secretory immunoglobulins. Along with this, currently NASA is also conducting research into probiotics aiming at human life in space.

\section{CONCLUSIONS}

Consumption of dietary probiotics does not have any major risk on the oral health of an individual. Prolonged administration of probiotics will result in an increase in the level of lactobacillus in the oral cavity, which may have beneficial effects on the host. As a precautionary measure there is a need to monitor the sugar level of the probiotic products as it is found that manufacturers add a lot of sugar in these products.

Financial or Other Competing Interests: None.

\section{REFERENCES}

[1] Pasteur L, Joubert JF. Charbon et septicemie. C R Soc Biol 1877;85:101-15.

[2] Gionchetti P, Amadini C, Rizzello F, et al. Probiotics- role in inflammatory bowel disease. Digestive and Liver Disease 2002;34 Suppl 2:S58-62.

[3] Lilly DM, Stillwell RH. Probiotics: growth-promoting factors produced by microorganisms. Science 1965;147(3659):747-8.

[4] Parker RB. Probiotics, the other half of the antibiotic story. Anim Nutr Health 1974;29:4-8.

[5] Furet JP, Kong LC, Tap J, et al. Differential adaptation of human gut microbiota to bariatric surgery-induced weight loss: links with metabolic and low-grade inflammation markers. Diabetes 2010;59(12):3049-57.

[6] Wu X, Ma C, Han L, et al. Molecular characterisation of the faecal microbiota in patients with type II diabetes. Curr Microbiol 2010;61(1):69-78.

[7] Schwiertz A, Taras D, Schäfer K, et al. Microbiota and SCFA in lean and overweight healthy subjects. Obesity (Silver Spring) 2010;18(1):190-5.

[8] Bjorksten B, Naaber P, Sepp E, et al. The intestinal microflora in allergic Estonian and Swedish 2-year-old children. Clinical and Experimental Allergy 1999;29(3):342-6.

[9] Kalliomaki M, Salminen S, Arvilommi H, et al. Probiotics in primary prevention of atopic disease: a randomized placebo-controlled trial. Lancet 2001;357(9262):1076-9.

[10] Majamaa H, Isolauri E. Probiotics: a novel approach in the management of food allergy. J Allergy Clin Immunol 1997;99(2):179-85.

[11] Rosenfeldt V, Benfeldt E, Nielsen SD, et al. Effect of probiotic Lactobacillus strains in children with atopic dermatitis. J Allergy Clin Immunol 2003;111(2):389-95.

[12] Klinder A, Forster A, Caderni G, et al. Fecal water genotoxicity is predictive of tumor preventive activities by inulin-like oligofructoses, probiotics (Lactobacillus 
rhamnosus and Bifidobacterium lactis), and their synbiotic combination. Nutr Cancer 2004;49(2):144-55.

[13] Bolognani F, Rumney CR, Pool-Zobel BL, et al. Effect of lactobacilli, bifidobacteria and inulin on the formation of aberrant crypt foci in rats. European Journal of Nutrition 2001;40(6):293-300.

[14] Roller M, Caderni G, Rechkemmer G, et al. Long term treatment with a prebiotic modulates the gut associated immune system of azomethane-treated F344 rats. Gastroenterology Research and Practice 2002:5-72.

[15] Puri S, Fernandez S, Puranik A, et al. Policy content and stakeholder network analysis for infant and young child feeding in India. BMC Public Health 2017;17(Suppl 2):461.

[16] Kogade P, Gaidhane AM, Choudhari S, et al. Socio-cultural determinants of infant and young child feeding practices in rural India. Medical Science 2019;23(100):1015-22.

[17] Thow AM, Karn S, Devkota MD, et al. Opportunities for strengthening infant and young child feeding policies in south Asia: insights from the SAIFRN policy analysis project. BMC Public Health 2017;17:404.

[18] Gaidhane AM, Sinha A, Khatib MN, et al. A systematic review on effect of electronic media on diet, exercise, and sexual activity among adolescents. Indian J Community Med 2018;43(Suppl 1):S56-65.

[19] Uddin S, Mahmood H, Senarath U, et al. Analysis of stakeholders networks of infant and young child nutrition programmes in Sri Lanka, India, Nepal, Bangladesh and Pakistan. BMC Public Health 2017;17:405.

[20] Balwani MR, Bawankule CP, Khetan P, et al. Awareness about kidney and its related function/dysfunction in school going children: a survey from the Central India. Saudi J Kidney Dis Transpl 2019;30(1):202-7.

[21] Mittal V, Jagzape T, Sachdeva P. Care seeking behaviour of families for their sick infants and factors impeding to their early care seeking in rural part of Central India. J Clin Diagn 2018;12(4):8-12.

[22] Orrhage K, Brisinar B, Nord CE. Effect of supplements with Bifidobacterium longum and Lactobacillus acidophilus on the intestinal microbiota during administration of clindamycin. Microb Ecol Health Dis 1994;7(1):17-25.

[23] Ujaoney S, Chandra J, Faddoul F, et al. In vitro effect of over-the-counter probiotics on the ability of candida albicans to form biofilm on denture strips. J Dent Hyg 2014;88(3):183-9.

[24] Wegner RD, Warner T, Roberts L, et al. Colonization of congenitally immunodeficient mice with probiotic bacteria. Infect Immun 1997;65(8):3345-51.

[25] Montalto M, Vastola M, Marigo L, et al. Probiotic treatment increases salivary counts of lactobacilli: a double-blind randomized controlled study. Digestion 2004;69(1):53-6.

[26] Sugano N. Biological plaque control: novel therapeutic approach to periodontal disease. J Oral Sci 2012;54(1):15.

[27] Meurman JH, Stamatova I. Probiotics: contributions to oral health. Oral Dis 2007;13(5):443-51.

[28] Capurso L. Probiotics and food tolerance. Probiotics and prebiotics and food intolerance. Allergy 2001;56(67):125-6.

[29] Koretz RL. Probiotics, critical illness and methodological bias. Nutr Clin Prac 2009;24(1):45-9.

[30] Ojetti V, Gigante G, Gabrielli M, et al. The effect of oral supplementation with Lactobacillus reuteri or tilactase in lactose intolerant patients: randomized trial. Eur Rev Med Pharmacol Sci 2010;14(3):163-70.

[31] Suvarna VC, Boby VG. Probiotics in human health. A current assessment. Current Science 2005;88(11):17448.

[32] Nagaraj T, Ravi B, Sankara SN, et al. Probiotics and oral health. Indian Acad Oral Med Radiol 2012;24(2):146-8. 